Review

\title{
A Multi-Disciplinary Examination of Psycho-Physiological Performance Among Youth and Junior Ball Sport Athletes: A Systematic Review
}

\author{
József Márton Pucsok ${ }^{1 *}$, Andrea Lentene Puskas ${ }^{1}$, Katalin Varga ${ }^{1}$, Gabriella Perenyi ${ }^{1}$, Eva Bacsne \\ Baba $^{2}$, Laszlo Balogh' \\ ${ }^{1}$ Institute of Sport Sciences, University of Debrecen, Debrecen, Hungary \\ ${ }^{2}$ Institute of Sporteconomics and Management, University of Debrecen, Debrecen, Hungary
}

\author{
Article Information \\ History: \\ Received: November 2, 2017 \\ Accepted: January 12, 2018 \\ Published: January 16, 2018 \\ Keywords: \\ Sport performance analysis \\ Talent development \\ Vienna test system \\ Youth ball sports
}

\begin{abstract}
Objectives: The primary purpose of our review was to systematically review the evolution of psycho-physiological performance analysis. Our secondary aim was to investigate the role of specific, multi-disciplinary performance indicators to identify future talents in the sport of soccer, handball and basketball.

Methods: A review protocol was set up in order to avoid individual bias and ensure an efficient searching prodedure. This enabled a search strategy to define which literature was to be included or excluded from our research. Certain inclusion criteria were identified, before performing a search using three electronic databases (Web of Science, ScienceDirect, and Google Scholar) and reference lists for English-language articles, published from the year 1980 till 2017.

Results: The comprehensive search yielded 82 documents to be relevant to our purpose of the study, according to our inclusion criteria criteria, 58 were fully-reviewed and referred to in our study. As we previously expected, the review of related literature resulted investigations involving mostly soccer and basketball players, studies conducted on handball players are significantly fewer. The majority of those studies examined physical performance characteristics via various field test.

Conclusions: We concluded that further analysis is necessary to identify relevant psycho-physiological performance indicators, in order to properly enhance sports performance in the younger athlete population and in general too. Vienna Test System protocols may offer a great potential to provide valuable information for athletes and their coaches to enhance athletic performance. Future research should focus on specifically identify Vienna Test System test protocols that may be linked to each particular sport.
\end{abstract}

\section{INTRODUCTION}

According to number of players, fans and audience soccer and basketball are among the top three most popular sports on the planet. The number of amateur and professional competitors participating in soccer and basketball are among the highest world-wide. Handball is also an Olympic ball sport with a long history, emerging popularity, specifically in our

*Correspondence: József Márton Pucsok, Institute of Sport Sciences, University of Debrecen, Egyetem tér 1, Debrecen, 4032 Hungary.

E-mail address: pucsok.jozsef@sport.unideb.hu region, in Europe. Enhancing physical abilities of young athletes in order to maximize performance in adult age is dated back in ancient times. Later, youth-oriented training programs have pointed out the misconception of dealing with adolescents as 'mini-adults' [1]. These early concepts have recognized the importance of physical growth and stature in order to deliver any training programs [2]. Since that, technology in sport has been rapidly developing, hightech performance analyzer equipments such as complex spiroergometric devices, high-speed motion capture cameras, computerized cognitive and psychomotor test batteries such as the Vienna Test System (VTS) have revolutionized analysis of sport performance. Team sports, especially ball 
sports are 'multi-factorial' sports, consist of a multitude of psycho-physiological performance parameters. There are a number of both field and laboratory tests which are advisable for both valid and reliable to monitor player's performance. Therefore the objective of this systematic review was to explore how a group of laboratory tests may be used to examine sports performance in junior athletes.

\section{METHODS}

\section{Review protocol}

We performed a systematic review of the available literature using Preferred Reporting Items for Systematic reviews and Meta-analyses (PRISMA) guidelines. In order to ensure the scientific integrity of our research, we defined four inclusion criteria: (1) providing data concerning psycho-physiological analyses of youth (age 13-15) and junior (age 16-18) ball sport athletes; (2) involving elite or sub-elite level soccer, handball or basketball players; (3) published as research articles or research notes; (4) studies only in English language. Exclusion criteria were the following: (1) ball sports other than soccer, handball or basketball; (2) no relevance with our research interest and (3) were conference abstracts or proceedings as previously recommended by Knipschild [3]. In order to provide the best possible academic relevance, we used specific search terms and strings. We chose, psycho-physiological analysis, performance analysis, VTS, soccer, basketball, handball, youth and junior ball sports terms to identify literature relevant to our research. The scoping study conducted prior to the actual reviewing process, supported our hypothesis, that only a few studies may be found involving youth and junior ball sport population. We created four search strings accordingly such as: psycho-physiological analysis and soccer; psycho-physiological analysis and basketball; psycho-physiological analysis and handball; VTS and sport. Our primary purpose was to provide a systematic overview of the evolution of performance analysis. The secondary aim was to investigate the role of specific, multi-disciplinary performance indicators to identify future talents in the sport of soccer, handball and basketball. Current examinations, undertaken here in University of Debrecen are going to investigate youth and junior (age 13-16) athletes participating in team-sports (soccer, basketball, and handball) and individual-sports (track and field, and judo). Our goal is to examine the relationship between various morphological, psycho-physiological performance indicators, also trying to draw practical sport-specific conclusions. We are positive that our future findings will facilitate the process of the development of individualized, specific training programs.

\section{Management of retrieved citations}

The number of total references retrieved was 920. We conducted a three-level screening of references monitoring their title, the abstract and finally the full-text of the selected papers. According to the inclusion and exclusion criteria, we conducted an automatic screening for duplicates, result of that, we removed 35 articles. This process was followed by further manual screening of references based on their titles and abstracts, another 803 articles were eliminated due to lack of relevance to our research interest. The remaining 82 full-text article was carefully reviewed, by the authors and co-authors, and 24 of those were further excluded from the study. Finally, we included 58 articles as a list of references to our systematic review.

\section{RESULTS}

\section{Analysis of physical performance profile in youth and junior ball sport athletes}

According to an individual's anthropometric measures, gender, and age, one may predict future performance. Success in the sport at any level also depends heavily on the level of physical fitness [4]. Team Sports (TS) indicate games played between two opposing teams. The players interact to achieve an objective that involves team members facilitating the movement of a ball or a similar item in accordance with a set of rules, to score points and to prevent the opposition from scoring [5]. Most of the performance analysis and studies referring to team ball sports have been overwhelmingly conducted in the sport of soccer. The reason is obvious; soccer is the number one, most widely popular sport on the planet. There is a continuous debate, whether results of field tests or laboratory analysis are the preferred indicators of performance in sport. When examining the same physiological parameter, for example, the maximal oxygen uptake, we would preferably choose a field test for the young or adolescent athletes. But scientists, practitioners in the sport have to be careful selecting the right test, in order to realistically assess a performance indicator. Soccer, like other ball sports, are predominantly characterized by, high-intensity intermittent movements. During a game, players demonstrate periods of short, intense anaerobic and longer duration aerobic effort. Typically, aerobic metabolism dominates in the recovery periods of the game [6].

There are certain advantages and disadvantages of field testing, which is the indirect and laboratory testing, which is the direct way of performance analysis. Field testing comes practically when testing high number of population or when necessary lab equipment is unavailable. In addition, these kinds of measurements, however, do not offer standardized conditions, may more realistically reflect the demands of a real match situation. There are certain, and obvious advantages of laboratory exercise testing, over field testing. One is reproducibility of a given analysis under standardized testing environment, so the entire testing procedure of an experiment or study may be duplicated [7]. VO2max is the index most widely used for assessing the aerobic fitness of soccer and ball sport players, and may be examined via various laboratory and field tests. In 2001, a study indicated that increasing aerobic capacity by $11 \%$ rised match intensity by $5 \%$ and distance completed in a match by $1800 \mathrm{~m}$ [8]. In a 
laboratory, it is far easier to provide standardized environment, which facilitates the direct evaluation of any physiological parameter. However, since soccer incorporates intermittent and rapid changes in the direction of movement, the nature of tests used to verify $\mathrm{VO} 2 \mathrm{max}$ in the laboratory conditions may differ from the actions observed in real match situation [9]. In soccer, the actual state of physical condition influences the execution of any technical or tactical skills [10,11]. Aerobic metabolism, dominating the game, average intensity is around the anaerobic lactate threshold. However, intensity during the game is constantly changing, requiring a high level of aerobic and anaerobic performance $[8,12]$. Running economy is another variable that influences aerobic endurance performance in a player. Improvement in running economy may result in a $5 \%$ or $1000 \mathrm{~m}$ increase in distance covered in a match. Elevated maximal oxygen uptake seems to increase both anaerobic threshold and running economy [11]. Assessment of an athlete's maximal oxygen consumption is possible involving either laboratory tests, or several kinds of field tests [13-15]. Researchers demonstrated that it is possible to determine VO2max with a soccer-specific test. Related literature suggests that none of the field tests have demonstrated a direct correlation with laboratory endurance tests $[11,12,16,17]$. The widely-used 'Shuttle Run Test (SRT)' introduced by [14] requires continuous, rather aerobic effort, while the 'Carminatti's test (TCar)' suggested by Carminatti et al. consist of intermittent type of movements. Reports regarding correlations between various field test results, such as the 'SRT and TCar tests' and the results of laboratory tests are rare and controversial [18-22]. In most cases, results of specific lab tests and field tests are not always correlating to each other. Researchers like Chamari et al., conducted the sport specific field test, called 'Bangsbo test', in thirty U19 elite soccer players [23]. After examining the elite junior athletes, they concluded that in this particular homogenous study population, the results of Bangsbo test did not provide a satisfactory estimate of the actual maximal oxygen consumption (VO2max). The results correlated only with the so-called vVO2max. The term vVO2max stands for the running pace, the velocity at maximal oxygen uptake, the point, where an individual's maximal oxygen uptake is reached [24]. There are other field tests such as the $20 \mathrm{~m}$ multi-stage endurance run, and the $20 \mathrm{~m}$ continuous progressive track run in order to estimate aerobic endurance. Players have to perform repetitive runs between two lines placed $20 \mathrm{~m}$ apart with a given speed, adjusted to an audio signal. At the end of each 3 min stage, a $90 \mathrm{~s}$ break is applied [25]. The ' $20 \mathrm{~m}$ continuous progressive track run test' is also a way to measure the value of maximal aerobic speed (vVO2max) [26]. This test is taken on a regular $400 \mathrm{~m}$ athletic track with cones placed every $20 \mathrm{~m}$. A pre-recorded soundtrack indicates when the player has to pass a cone to keep up with the exact speed. Each stage is separated by a longer sound. The beginning stage is set at 8 $\mathrm{km} / \mathrm{h}$ with subsequent increase of $0.5 \mathrm{~km} / \mathrm{h}$ per 1 -min stage. The test is terminated when the athlete is unable to continue with the pre-set running speed. Maximal aerobic capacity or
vVO2max corresponds the speed $(\mathrm{km} / \mathrm{h})$ measured at the last completed stage.

Most of the previously published studies, which investigated the physical fitness of elite ball sport athletes used different field tests. These studies are often contradicting and inconclusive regarding the role and importance of different physical related to the playing level and position in elite ball sports, especially in handball [27]. Some studies concluded differences in variables such as the velocity of a throw [2831], dynamical characteristics and the height of the jumps [32], absolute values of bench press and half squat [29]. While other researchers such as $[29,33]$ suggested no significant differences in sprinting and jumping ability, in bench pressing or squatting. In contrast, observed a strong relationship between morphological parameters, performance characteristics and playing position [34]. In the recent years, researchers such as monitored physical performance profile by using incremental spiroergometric test protocols as well [31,34-36]. The comprehensive search of the related databases resulted studies, which evaluated mostly senior handball players, members of national teams, not youth or juniors. Some researchers conducted an in-depth match analysis, and later investigated the effect of 8-week plyometric training in elite adolescent handball players $[37,38]$. According to the results of our database searches, studies conducted in youth player population are very rare.

In the sport of basketball related literature have shown that physiological demand varies regarding to the position of the player [39]. Researchers compared physical characteristics of players with different age and position [40]. The authors observed differences related to physical condition in that particular study population. Other researchers such as Jeličic et al. were trying to establish relationship between morphological status, situational and functional parameters of elite junior players [41]. There are only a very few studies, which provide performance profiles across anthropometric and physiological variables for a large group of elite level youth and junior basketball players [42]. Among those conducted a study measuring anthropometric and physiological characteristics of 125 male and 123 female junior basketball players participating at the Australian Under 16 championships in 1998 [43]. The author observed differences across playing positions with regard to body composition measures (height, and body mass and arm span in particular) and to a lesser degree speed and agility. The researcher concluded, that the level of players may be characterized by a number of body composition (height, sitting height, and arm span) and physiological variables (speed, agility, vertical jump, basketball throw, and aerobic endurance). The top ranked, center players were difficult to evaluate based on test performance. In conclusion, some of the treadmill running tests applied in ball sports may not provide an accurate estimate of maximal aerobic fitness because soccer, basketball and handball consist of multi-directional movements. Researchers such as offered a solution to these phenomena, who assessed the physiological capacity and various performance characteristics using heart-rate (HR) monitors and computerized 
movement and technical match analysis $[35,44,45]$.

\section{Analysis of psycho-physiological performance profile in youth and junior ball sport athletes}

Psychological profiling of athletes, players of thedifferent level has been always a key part of sport performance evaluation. It is thought, that talented athletes may own personality characteristics that enhance motor learning, successful participation in a competition and the training process itself. There has been an on-going dispute among participants, professionals in sport, whether 'gifted' and less talented players may be distinguished on the basis of their 'psychological makeup'. Researchers have not yet fully identified specific personality characteristics, or an overall psychological profile, that are predictably associated to success in sport. According to studies conducted by Williams \& Reilly, and Morris, there isn't any obvious or consistent relationship between personality and expertize [46,47]. Researchers have been trying to identify a single psychological inventory to help select potential players, but it is hardly likely that any single approach would be able to predict future talents. Predicting performance is even more complicated in ball sports. Most previous studies on talent identification and selection in soccer mainly focused on differences between elite, subelite and/or recreational youth players, or examined a homogeneous group of players who differed in biological maturation. Other studies indicated, that more mature selection groups with higher morphological measures would outperform the late maturers on the fitness tests, but not on the specific and non-specific motor coordination tests $[48,49]$.

\section{Application of VTS in sport}

However, some parameters such as perception and decision making may be useful in predicting sport performance. The combination of sport specific tests, laboratory analyzes and measures of perception, decision making via for example the VTS would be worthwhile avenues for future research [43]. The VTS is a computerized collection of assessments and tests that can be used alone or combined with a test battery. The test is administered and scored by computer [50]. Schuhfried provided a complete description of psychological assessment of the VTS. The author listed ten different test batteries such as the 'Two Hand Coordination Test (2HAND)', the 'Coloured Progressive Matrices test (CPM)' etc. may be used in main areas such as psycho-clinical, psycho-neurological, work and occupation, sports and leisure. Two variables the speed of perception and the speed and accuracy of reaction are critical elements in ball sport performance. In the 'Peripheral Perception Test (PP)', participants are asked to detect a vertical light bar which turns up intermittently on a special display. The participants of the 'Reaction Test (RT)' are requested to press a response key as soon as they notice the so called critical stimulus. A comprehensive search of related databases resulted over twenty studies related to the topic. The studies investigating this area have been split into two major categories. The first group of studies investigate two different groups of people: athletes and non-athletes; athletes from the same sport who are at different levels; and athletes from various sports. The second category of studies, aimed to examine the effect of a designated parameter on the performance of athletes in various VTS tests. To the author's knowledge, there are only very few studies found, involving youth and junior athletes, especially in ball sport, utilizing VTS test protocols in the evaluation of ball sport performance [51,52]. A recent comprehensive review by Ong, demonstrated that VTS has a great potential to provide valuable information for athletes and their coaches to enhance sport performance [53]. Future research should focus on specifically identify VTS test protocols that are relevant to each particular sport.

\section{CONCLUSIONS}

Sport performance is a complex phenomenon depends not only on physical capacity but various psychological variables too. Body composition, endurance, muscle strength, power and agility and also perceptual speed and reaction capacity variables providing important information for sport professionals when detecting, identifying and finally developing talents in sport. Nowadays, professionals in sport agree, that the development of any performance-related abilities does not generate the same effect or adaptation in players at different ages [54]. Several investigations among those suggest, that the stimulus triggered by the maturational development of the players, and the physical components of a given training session should be incorporated with technical and tactical preparation $[55,56]$. In a pre-planned training process, participants in sport need to evaluate physiological response to each game situation, and also to develop a behaviour that may be transferred to competition, integrating all the qualities and factors that improve the capacity to play. In the 21 st century, coaches ability to detect and identify talents is still crucial, instead of selecting the high performers in their childhood, they should target potential young talents, who possess certain skills and attributes to become elite athletes in the future. Young talents may have lower morphological measures, not as skillful yet, but demonstrate a high level of coachability, sensitivity to training, and perhaps most importantly the motivation to learn. Identifying talent requires a complex evaluation of all the physical, physiological, psychological, technical, and hereditary components, and capabilities of an athlete [57]. In 2015, the International Olympic Committee (IOC) issued a common statement on youth athletic development. Youth athlete development is an individually unique, based on constantly changing physical growth, biological maturation and behavioural development, and therefore it must be considered individually. According to Bergeron et al., "a rationale for performance analysis the assessment and monitoring of youth athletes might include purportedly identifying talent, predicting future performance, determining strengths and weaknesses, informing the selection process, evaluating the effectiveness of training programmes, 
monitoring current health and performance, motivating the athlete, enhancing the athlete's (and coach's) understanding of the demands of the sport, and improving present and future performance" [58]. Therefore, it is essential that the physical and mental preparation of athletes should be focused on the specific skills required for each playing position. Areas of future research may include interaction between the anthropometrical, physiological and psychological components of training. Other interesting topics are the periodization of training-the balance between training stress and recovery in particular sports and the examination of hereditary factors and genetic predispositions in sport performance.

\section{ACKNOWLEDGEMENTS}

This study was supported by the Economic Development and Innovation Operational Programme (grant number: 2014-2020 - GINOP 2.3.2.-15-2016-00047).

\section{Conflicts of interest}

The authors declare no conflict of interest.

\section{REFERENCES}

1. Bompa TO. Total Training for Young Champions. Champaign, IL, USA. Human Kinetics. 2000, p 21-31.

2. Faigenbaum AD, Kraemer WJ, Blimkie CJ et al. Youth resistance training: Updated position statement paper from the National Strength and Conditioning Association. J Strength Cond Res, 2009; 23: S60-79.

3. Knipschild P. Some examples of systematic reviews. In I Chalmers \& D. G. Altman (Eds.), Systematic reviews. London: BMJ Publishing Group. 1995, p 9-16.

4. Williams C. ABC of sports medicine. Assessment of Physical Performance. BMJ, 1994; 309: 180-184.

5. Garganta J. Trends of tactical performance analysis in team sports: bridging the gap between research, training and competition. Rev Por Ci Desp, 2001; 9(1): 81-89.

6. Higino WP, Sorroche A de S, de Mattos Falqueiro PG, Suzuki Lima YC, Higa CL. Determination of Aerobic Performance in Youth Soccer Players: Effect of Direct And Indirect Methods. J Hum Kinet, 2017; 56: 109-118.

7. Fletcher GF, Ades PA, Kligfield P. et al. Exercise standards for testing and training- a scientific statement from the American Heart Association. Circulation. 2013; 128: 873-934.

8. Helgerud J, Engen LC, Wisloff U, Hoff J. Aerobic endurance training improves soccer performance. Med Sci Sports Exerc, 2001; 33(11): 1925-1931.

9. Drust B, Atkinson G, Reilly T. Future perspectives in the evaluation of the physiological demands of soccer. Sport Med, 2007; 37(9): 783-805.

10. Bangsbo J, Mohr M, Krustrup P. Physical and metabolic demands of training and match-play in the elite football player. J Sport Sci, 2006; 24 (7): 665-674.

11. Hoff J, Wisløff U, Engen LC, Kemi OJ, Helgerud J.
Soccer specific aerobic endurance training. British J Sport Med, 2002; 36: 218-221.

12. Bangsbo J, Lindquist F. Comparison of various exercise tests with endurance performance during soccer in professional players. Int J Sports Med, 1992; 13:125-132.

13. Leger L, Boucher R. An indirect continuous running multistage field test: the Universite' de Montreal track test. Can J Appl Sport Sci, 1980; 5: 77-84.

14. Léger LA, Lambert J. Maximal 20m Shuttle Run Test to predict VO2max. Eur J Appl Physiol Occup Physiol, 1982; 49(1): 1-12.

15. Léger LA, Mercier D, Gadoury C, Lambert J. The multistage 20 metre shuttle run test for aerobic fitness. J Sport Sci, 1988; 6: 93-101.

16. Reilly T, Bangbso J, Franks A. Anthropometric and physiological predispositions for elite soccer. J Sport Sci, 2000; 18: 669-683.

17. Mujika I, Padilla S, Ibañez J, Izquierdo M, Gorostiaga E. Creatine supplementation and sprint performance in soccer players. Med Sci Sports Exerc, 2000; 32: 518-525.

18. Carminatti LJ, Lima AES, Oliveira, FR. Aerobic fitness in intermittent sports - Evidence of construct validity and results in incremental test with pause. Rev Brasil Fisiol Exerc, 2004; 3(1): 1-20.

19. Aziz AR, Tan FHY, Teh KC. A Pilot Study Comparing Two Fields Tests with the Treadmill Run Test in Soccer Players. J Sport Sci Med, 2005; 4 (2): 105-112.

20. Denadai BS, Higino WP, Faria RA, Nascimento EP, Lopes EW. Validity and reproducibility of the blood lactate response during the shuttle run test in soccer players. Rev Brasil Ci Mov, 2002; 10(2): 71-78.

21. Silva JF, Guglielmo LGA, Carminatti LJ, Oliveira FR, Dittrich N, Paton CD. Validity and reliability of a new field test (Carminatti's test) for soccer players compared with laboratory-based measures. J Sport Sci, 2011; 29(15): 1621-1628.

22. St Clair Gibson A, Broomhead S, Lambert MI, Hawley JA. Prediction of maximal oxygen uptake from 20-m shuttle tun as measured directly in runners and squash players. J Sport Sci, 1998; 16(4): 331-335.

23. Chamari K, Hachana Y, Ahmed YB, et al. Field and laboratory testing in young elite soccer players. Brit J Sport Med, 2004; 38 (2): 191-196.

24. Billat VL, Koralsztein JP. Significance of the Velocity at VO 2max exhaustion at this velocity. Sports Med, 1996; 22: 90-108.

25. Gonaus C, Müller E. Using physiological data to predict future career progression in 14- to 17-year-old Austrian soccer academy players, J Sport Sci, 2012; (30) 15: 1673-1682.

26. Chtara M, Chamari K, Chaouachi MA, et al. Effects of intra-session concurrent endurance and strength training sequence on aerobic performance and capacity. Brit J Sport Med, 2005; 39: 555-560.

27. Haugen T, Tonnessen E, Seiler S. Physical and physiological characteristics of male handball players: Influence of playing position and competitive level. J Sports 
Med Phys Fit, 2016; 56 (1-2): 19-26.

28. Bayios IA, Anastasopoulou EM, Sioudris DS, Boudolos KD. Relationship between isokinetic strength of the internal and external shoulder rotators and ball velocity in team handball. J Sports Med Phys Fit, 2001; 41: 229-235.

29. Gorostiaga EM, Granados C, Ibánez J, Izquierdo M. Differences in physical fitness and throwing velocity among elite and amateur male handball players. Int J Sports Med, 2005; 26: 225-232.

30. Granados C, Izquierdo M, Ibánez, J, Ruesta, M, Gorostiaga EM. Are there any differences in physical fitness and throwing velocity between national and international elite female handball players? J Strength Cond Res, 2013; 27: 723-732.

31. Wagner H, Buchecker M, von Duvillard SP, Müller E. Kinematic comparison of team handball throwing with two different arm positions. Int J Sports Phys Perf, 2010; 5: 469-483.

32. Krüger K, Pilat C, Ueckert K, Frech T, Mooren FC. Physical performance profile of handball players is related to playing position and playing class. J Strength Cond Res, 2014; 28: 117-125.

33. Chaouachi A, Brughelli M, Levin G, Boudhina NB, Cronin J, Chamari K. Anthropometric, physiological and performance characteristics of elite team-handball players. J Sport Sci, 2009; 27: 151-157.

34. Buchheit M, Al Haddad H, Millet GP, Lepretre PM, Newton MM, Ahmaidi S. Cardiorespiratory and cardiac autonomic responses to 30-15 intermittent fitness test in team sports players. J Strength Cond Res, 2009; 23(1): 93-100.

35. Póvoas SC, Seabra AF, Ascensão AA, Magalhães J, Soares JM, Rebelo AN. Physical and physiological demands of elite team handball. J Strength Cond Res, 2012; 26: 3365-3375.

36. 3Michalsik LB, Aagaard P, Madsen K. Physiological capacity and physical testing in male elite team handball. J Sports Med Phys Fit, 2015; 55(5):415-429.

37. Ignjatovic AM, Markovic ZM, Radovanovic DS. Effects of 12-week medicine ball training on muscle strength and power in young female handball players. J Strength Cond Res, 2012; 26: 2166-2173.

38. Chelly MS, Hermassi S, Aouadi R, et al. Match analysis of elite adolescent team handball players. J Strength Cond Res, 2011; 25: 2410-2417.

39. Rodriguez-Alonso M, Fernandez-Garcia B, Perez-Landaluce J, Terrados N. Blood lactate and heart rate during national and international women's basketball. J Sports Med Phys Fit, 2003; 43(4): 432-436.

40. Abdelkrim NB, Chaouachi A, Chamari K, Chtara M, Castagna C. Positional role and competitive-level differences in elite-level men's basketball players. J Strength Cond Res, 2010; 24 (5): 1346-1355.

41. Jeličic M, Sekulic D, Marinkovic M. Anthropometric characteristics of high level European junior basketball players. Coll Antrop, 2002; 26: 69-76.
42. Ostojic SM, Mazic S, Dikic N. Profiling in basketball: Physical and physiological characteristics of elite players. J Strength Cond Res, 2006; 20(4): 740-744.

43. Hoare DG. Predicting success in junior elite basketball players--the contribution of anthropometric and physiological attributes. J Sci Med Sport, 2000; 3 (4): 391-405.

44. Michalsik LB, Aagaard P. Physical demands in elite team handball: Comparisons between male and female players. J Sports Med Phys Fit, 2015; 55(9):878-91.

45. Michalsik LB, Aagaard P, Madsen K. Locomotion characteristics and match-induced impairments in physical performance in male elite team handball players. Int J Sports Med, 2013; 34 (7): 590-599.

46. Williams AM, Reilly T. Talent identification and development in soccer. J Sport Sci, 2000; 18(9): 657-667.

47. Morris T. Psychological Skills Training: An Overview, 2nd edn. Leeds: British Association of Sport and Exercise Sciences. 1997.

48. Serrano J, Shahidian S, Sampaio J, Leite N. The Importance of Sports Performance Factors and Training Contents From the Perspective of Futsal Coaches. J Hum Kinet, 2013; 38: 151-160.

49. Sanz A, Guerrero A. Futsal: meaningful tasks for integrated training. From traditional to modern training. Madrid (Spain). Ed. Editorial Gymnos. 2005.

50. Schuhfried G. Vienna test system: Psychological assessment. Moedling, Austria: Schuhfried. 2013.

51. Zisi V, Giannitsopoulou E, Vassiliadou O, Pollatou E, Kioumourtzoglou E. Performance level, abilities and psychological characteristics in young junior rhythmic gymnasts: the role of sport experience. Int Q Sport Sci, 2009; 4: 1-13.

52. Zwierko T, Glowacki T, Osinski W. The effect of specific anaerobic exercises on peripheral perception in handball players. Kines Sloven, 2008; 14(1): 68-76.

53. Ong CH N. The use of the Vienna Test System in sport psychology research: A review. Int Rev Sport Exerc Psychol, 2015; 8(1): 204-223.

54. Leite N, Coelho E, Sampaio J. Assessing the Importance Given by Basketball Coaches to Training Contents. J Hum Kinet, 2011; 30: 123-133.

55. Helsen W, Hodges N, Van Winckel J, Starkes J. The roles of talent, physical precocity and practice in the development of soccer expertise. J Sport Sci, 2000; 18: 727-736.

56. Glamser F, Vincent J. The relative age effect among elite american youth soccer players. J Sport Behav, 2004; 27: 31-38.

57. O'Sullivan J. Our Biggest Mistake: Talent Selection Instead of Talent Identification. Published in Coaching, Problems in Youth Sports, Sports Parenting. 2013.

58. Bergeron MF, Mountjoy $\mathrm{M}$, Armstrong $\mathrm{N}$, et al. International Olympic Committee consensus statement on youth athletic development. Brit J Sport Med, 2015; 49: 843-551. 